\title{
Anosmia and ageusia: a piece of the puzzle in the etiology of COVID-19-related transitory erectile dysfunction
}

\author{
R. Bertolo ${ }^{1}$ (D) C. Cipriani ${ }^{1} \cdot$ P. Bove ${ }^{1,2}$
}

Received: 6 January 2021 / Accepted: 19 January 2021 / Published online: 29 January 2021

(C) Italian Society of Endocrinology (SIE) 2021

\section{Letter to the editor-Re: Sansone A, Mollaioli D, Ciocca G, et al. Addressing male sexual and reproductive health in the wake of COVID-19 outbreak. J Endocrinol Invest. 2020 Jul 13;1-9. https://doi.org/10.1007/ s40618-020-01350-1.}

In late December 2019, a cluster of unexplained cases of viral pneumonia occurred in Wuhan, China. On February 11, 2020, the World Health Organization officially named the disease caused by the 2019-nCoV (nowadays renamed "SARS-CoV-2") as coronavirus disease (COVID-19), with its clinical presentation including a severe form of acute respiratory syndrome. From the initial cluster, it spread into plenty of Countries with a variety of symptoms described in the typical COVID-19 [1].

Despite several thousand casualties, the majority of people diagnosed with COVID-19 have survived, with a small amount of them admitted into intensive care units (ICU).

Erectile dysfunction (ED) has been described to be a possible consequence of COVID-19 for survivors. While being a trivial matter while admitted into ICU, ED represents a worrying phenomenon for a large part of affected patients. As such, it has been reported how sexual activity plays a protective effect on the quarantine-related plague of anxiety and mood disorders during the COVID-19 pandemic [2].

The effort by Sansone and colleagues offered a literature review of the mechanisms involved in the development of ED in COVID-19 survivors [3].

Many causes have been addressed able to contribute to the potential onset of ED: among them endothelial dysfunction,

$\triangle$ R. Bertolo

riccardobertolo@hotmail.it

1 Urology Department, San Carlo Di Nancy Hospital, GVM Care and Research, Via Aurelia 275, 00165 Rome, Italy

2 Department of Surgery, Urology Unit, Tor Vergata University of Rome, Rome, Italy subclinical hypogonadism, psychological distress and impaired pulmonary hemodynamics have been mentioned. Additionally, the authors underlined that COVID-19 might exacerbate pre-existing cardiovascular conditions, further increasing the risk of ED. Finally, they have recommended careful investigation of testicular function in COVID-19 patients due to the unclear association with testosterone deficiency and the possible consequences for reproductive health.

Herein, we would like to stress a concept that is missing. In a non-negligible number of patients affected by COVID19 , especially in those patients who know first-hand the disease as a paucisymptomatic flu, anosmia and ageusia can represent the first or only symptomatology present [4]. Recent studies have suggested that angiotensin-converting enzyme 2 receptors are strongly expressed on the nasal mucosa, suggesting the fact that SARS-CoV-2 might exert a toxic action on the multiple central nervous system's sites, including the olfactory bulb, for instance [5]. Moreover, the relationship between anosmia and hypogonadism as observed in the Kallmann syndrome is well-established in literature.

Anosmia represents an issue for male patients' sexual and reproductive health. The smell is the most used sense in the animal world and considered as the "sexual appeal of men".

Sex pheromones have evolved in many species. They are associated with signaling mating behaviors or dominance. The odors released can be seen as a favorable trait selected by either the male or female leading to attraction and copulation. Chemical signaling is also used to avoid inbreeding, thus preventing genetically transmitted diseases. Although human sex pheromones have never been isolated, and human beings are highly dependent upon visual cues, we believe that smells play a role in sociosexual behaviors when in close proximity [6]. It is through smells that the arousal mechanism in the brain is activated, that the olfactory alchemy between the partners can be triggered, and that passion can be ignited. The smell is perceived without the 
mediation of rationality, so it is perfect for fixing memories linked to emotionality and for igniting the most ancestral passion. The scent of sex is a powerful aphrodisiac and a way for recognizing each other between partners.

On the other hand, the taste is said to somehow anticipate sex. Thus, ageusia represents another major issue for men's sexual health. Taste is the sense through which to savor intimacy better. With a kiss, we can first of all relish the "taste" of a partner, allowing the discovery of new flavors, but also stimulate and recognize the partner. The kiss is therefore the "testimonial" of this sense, a real source of intimate pleasure, the act that ignites desire: saliva is rich in testosterone, the male hormone that enhances the sexual desire, therefore the exchange through the kiss stimulates the taste buds, sending exciting messages to the brain.

Together with the undoubted matters in carrying out sexuality in COVID-19 patients experiencing transitory anosmia, ageusia, and subsequent ED, the reader should bear in mind that such symptoms could be themselves the possible clinical consequences of an underlying COVID-19-triggered neurological disease. An increasing body of evidence is pointing out how the recently discovered SARS-CoV-2 has an important neurotropism that could actually enhance the development of neurological disorders that could be the intrinsic cause of ED.

We felt such insights of paramount importance in raising the awareness on the fact that anosmia and ageusia might play an important role in the etiology of a COVID-19-related temporary erectile dysfunction.

Further investigations are warranted to better explore this fascinating field.

\section{Compliance with ethical standards}

Conflicts of interest On behalf of all authors, the corresponding author states that there is no conflict of interest.

\section{References}

1. Rocco B, Sighinolfi MC, Sandri M et al (2021) The dramatic COVID 19 outbreak in Italy is responsible of a huge drop of urological surgical activity: a multicenter observational study. BJU Int 127(1):56-63. https://doi.org/10.1111/bju.15149 (Epub 2020 Oct 19)

2. Mollaioli D, Sansone A, Ciocca G et al (2021) Benefits of sexual activity on psychological, relational, and sexual health during the COVID-19 breakout. J Sex Med 18:35-49

3. Sansone A, Mollaioli D, Ciocca G et al (2020) Addressing male sexual and reproductive health in the wake of COVID-19 outbreak. J Endocrinol Invest. 2020;13:1-9. https://doi.org/10.1007/ s40618-020-01350-1 (online ahead of print)

4. Vaira LA, Salzano G, Deiana G, De Riu G. Anosmia and ageusia: common findings in COVID-19 patients. Laryngoscope 2020;130(7):1787. https://doi.org/10.1002/lary.28692. Epub 15 Apr 2020

5. Dolatshahi M, Sabahi M, Aarabi MH. Pathophysiological clues to how the emergent sars-cov-2 can potentially increase the susceptibility to neurodegeneration. Mol Neurobiol. 2021;1-16. https:// doi.org/10.1007/s12035-020-02236-2 (online ahead of print)

6. Bernstein C, Bernstein H (1997) Sexual communication. J TheorBiol 188(1):69-78. https://doi.org/10.1006/jtbi.1997.0459

Publisher's Note Springer Nature remains neutral with regard to jurisdictional claims in published maps and institutional affiliations. 